\title{
Evaluation of a new vine trellis system called Ramé $I$ Evaluación de un nuevo sistema de conducción de la Vid denominado Ramé
}

\author{
Bergas Valeria $^{1, a}$, Rodríguez José ${ }^{1}$, Galarraga Ignacio $^{1}$, Vila Hernán ${ }^{2}$, Carrillo Natalia ${ }^{1}$, Corvalán Andrea ${ }^{1}$, \\ and Gómez Francisco ${ }^{1}$ \\ ${ }^{1}$ Cátedra de Viticultura, Facultad de Ciencias Agrarias, UNCuyo, Almirante Brown 500, Mendoza, Argentina, cp 5507 \\ ${ }^{2}$ INTA, Instituto Nacional de Tecnología agropecuaria. San Martín y Araoz, Lujan de Cuyo, Mendoza
}

\begin{abstract}
You should leave $10 \mathrm{~mm}$ of space above the abstract and $15 \mathrm{~mm}$ after the abstract. The heading Abstract should be typed in bold 9-point Arial. The body of the abstract should be typed in normal 9-point Times in a single paragraph, immediately following the heading. The text should be set to 1.15 line spacing. The abstract should be centred across the page, indented $15 \mathrm{~mm}$ from the left and right page margins and justified. It should not normally exceed 200 words. Under this problematic context, several growers in our area proposed alternatives to conduction systems. An innovation was the system of divided canopy "Ramé", developed by the local viticulture Luis Alberto Ramero. This system was attempted to combine high yield with high quality grape, machanize harvest and good cinditions of canopy microclimate. It's a system of vertical shoot position, with its canopy divided into two planes. These systems are characterized, in our region, by a low foliar surface exposed per hectare, a relatively low productivity (in the case of the VSP) or difficulty to mechanize the harvest (in the case of the Parral). The objective of this study was to evaluate the ecophysiology of Rame system, determining if it behaves differently when it is planted with one or two plants by post (Rame single and double). Also the two modalities of Rame were compared with the trellis in VSP. Since this latter system is not included in the statistical, but planted design on the side of the test, the comparison kept a descriptive character. For the Rame we established an experimental trial with statistical design, with two treatments in trellis-training system. A treatment consisted in planting one vine per post, called simple Rame (RS) and two vines per post, called double Rame (RD), leaving in this way different number of plants per unit area (1600 plantas / has and 3200 plantas / has respectively). Nevertheless, regardless of the number of plants per hectare, Rame system maintain high values of anthocyanins (1, $2 \mathrm{mg} / \mathrm{Kg}$ of grapes) and IPT (43) with high production (152 qq / has). This fact, added to that the system has potential for mechanization, converts Rame in a trellis system with high potential for vine growth in the viticulture regions. The trellis VSP behaved less productive ( $86 \mathrm{qq} / \mathrm{has}$ ), and at the same time obtained lower values of qualitative components such as anthocyanins (1.04 $\mathrm{mg} / \mathrm{Kg}$ of grapes) and IPT (35).
\end{abstract}

\begin{abstract}
Resúmen. Los sistemas de conducción más difundidos en Argentina son los de canopia simple, tanto de desarrollo horizontal, caso de los parrales, como de desarrollo vertical, en los espalderos. En la actualidad predomina el parral, existe un crecimiento significativo de espalderos altos en superficie cultivada. Bajo este contexto problemático, varios viticultores de nuestra zona propusieron modificaciones a los sistemas de conducción. Una innovación fue el sistema de canopia dividida "Ramé", desarrollado por el viticultor mendocino Luis Alberto Ramero. Lo que este viticultor buscaba era poder tener mayores rendimientos (equiparables a los de un parral), manteniendo una alta calidad enológica de uva. También, poder colocar tela antigranizo de manera económica y mecanizar la cosecha mediante una máquina diseñada ad hoc que sacude los cordones secundarios. El objetivo de este trabajo fue evaluar ecofisiológicamente el sistema Ramé, determinando si se comporta en forma distinta cuando es plantado con una o dos plantas por poste (Ramé simple y doble). Para su evaluación se realizaron distintas mediciones, dentro de ellas nos enfocamos en superficie foliar Total por producción de uva, producción por planta y composición de Antocianos e IPT (Índice de polifenoles totales). No se encontraron diferencias significativas en ninguna de las variables medidas para los dos tratamientos. Sí se pudo observar que el sistema, independientemente del número de plantas por has, mantiene valores altos de Antocianos (1.2 mg/Kg de uva) e IPT (43) con alta producción (152 qq/Ha). Esto sumado a que el sistema tiene posibilidades de mecanización, se puede concluir que es un sistema de conducción con altas posibilidades de crecimiento en el medio.
\end{abstract}

\footnotetext{
a e-mail: vbergas@fca. uncu.edu.ar 


\section{Introducción}

La superficie cultivada con uvas en todo el país alcanzó en 2011 un total de 218.421 hectáreas contra unas

202.146 hectáreas existentes en 1990 (INV, 2011). Si bien la superficie implantada con viñedos no ha tenido un fuerte incremento, si se observa en variedades de alta ca- lidad enológica (varietales) un crecimiento del $78 \%$ en los últimos 20 años, como consecuencia de una reconversión productiva del sector, lo que representa unas 130 mil hectáreas en la actualidad contra las 72 mil existentes en 1990. En Mendoza la superficie de uvas de alta calidad enológica tuvo un importante incremento, pasando de 57.000 ha en el año 1990, a 101.500 ha en el año 2011, representando un crecimiento del $78 \%$ en dos décadas (INV, 2011).

Las variedades tintas son las que ha determinado el salto de calidad, dado que en el lapso de 20 años la implanta- ción de uvas tintas de alta calidad enológica ha sido significativamente alta, mientras que la superficie im- plantada con blancas con atributos enológicos de alta ca- lidad muestran una caída del 10\% (INV, 2011).

En Mendoza existen 11.899 ha, concentrando la mayor superficie el departamento Luján de Cuyo con 2.306 ha, lo que representa el $19,38 \%$ del total de la variedad en la provincia y el 14,59\% del total del departamento. Esta variedad también tiene importancia por su participación relativa en los departamentos Maipú con 1.589 ha, San Rafael con 1.282 ha, Tunuyán con 1.268 ha y San Martín con 1.002 ha. (INV, 2015).

En la vid, el sistema de conducción puede definirse como la forma y disposición que se da a las diferentes partes de la planta (Reynolds, 2009). Existen diversos tipos de estructuras de sostén que condicionan la altura del tronco, la dirección de los brazos, los elementos de poda y la exposición del follaje a la luz solar (Carbonneau, 1985).

La expresión de la tipicidad del vino depende del sistema de conducción, en la medida en que las prácticas de cultivo modifican el desarrollo natural de las plantas e influyen sobre la captación de luz (Carbonneau, 2007). A través del tiempo se han propuesto diferentes arquitecturas de canopia con el fin de lograr un mayor aprovechamiento de la luz solar y modular el vigor de las plantas (Carbonneau, 2007). El pionero en estudios sobre sistemas de conducción, Nelson Shaulis, creador del Geneva Doble Cortina (GDC) en Estados Unidos en la década de 1960 , fue el primero que percibió la importancia de estos aspectos. Su mérito consistió en romper el esquema tradicional de cultivo de la vid en espaldera o vaso, al dividir la canopia en dos planos verticales de vegetación descendente. Esto duplicó la superficie fotosintética de la planta y permitó una mayor aireación y mejor penetración de la luz en la zona de los racimos. Con esto consiguió un aumento perceptibe en la cantidad y calidad de uva cosechada, con vides vigorosas y baja densidad de plantas por hectárea (Shaulis et. al., 1966). Otro sistema de conducción de canopia dividida en dos planos fue el propuesto por Carbonnea en 1985 llamado Lira; que a diferencia del GDC, los brotes crecen hacia arriba.

Un modelo propuesto por Carbonneau (Carbonneau 1980, 1995), permite evaluar para todas las formas de arquitecturas de canopia, la superficie foliar expuexta (SFE). Es una estimación basada en un esquema

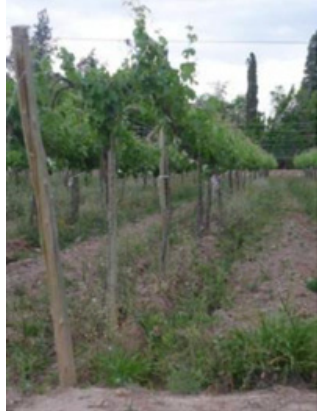

(a)

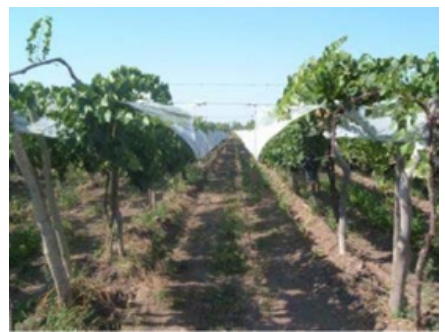

(b)

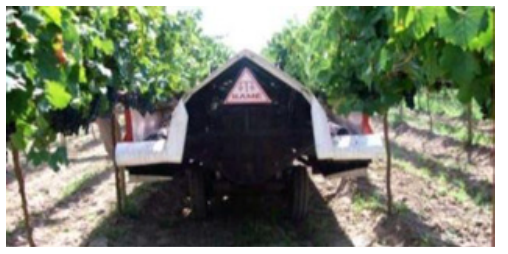

(c)

Figura 1.

representativo de la vegetación y de la superficie foliar por unidad de superficie de suelo que es capaza de optimizar el potencial fotosintético. Este modelo de SFE ha sido utilizado para evaluar el potencial de un viñedo (Carbonneau et al., 2007), y ha sido validado a través de análisis sensorial de vinos. Las variables del modelo que predicen las variables de calidad son: SFE y producción de uva P (SFE/P) (Carbonneau, 2010).

Otra invención, de un productor Mendocino, que respetó el principio de la canopia dividida, fue el Sistema Ramé desarrollado por el viticultor mendocino Luis Alberto Ramero (Zalazar, 2011). Con este sistema se ha intentado combinar alta producción, con alta calidad de uva y cosecha mecanizable. Este sistema se encuentra en el medio desde el año 2005. Se ha difundido en distintas zonas productivas de Mendoza y San Juan. Se ha probado con distintas variedades, algunas más productivas (Torrontes Riojano, Bonarda), y otras menos productivas (Malbec, Cabernet Sauvignon), pero todas con alta calidad enológica.

Se trata de un sistema de canopia vertical, dividida en dos planos, en el que, a diferencia del GDC, los brotes crecen hacia arriba. Los cordones, sobre los cuales apoyan los pitones, están en un brazo secundario, y estos brazos se desarrollan a partir de brazos primarios que se extienden primero horizontalmente alejándose del tronco de la planta y luego en forma descendente. En total la planta queda formada con dos brazos secundarios y cuatro cordones con pitones (Fig. 1(b)). Lo que este viticultor buscaba era poder tener mayores rendimientos (equiparables a los de un parral), manteniendo una alta calidad enológica de uva. También, poder colocar tela antigranizo de manera económica (Fig. 1(b)) y mecanizar la cosecha mediante una máquina diseñada ad hoc que sacudía los cordones secundarios (Fig. 1(c)).

El objetivo de este trabajo fue evaluar ecofisiológicamente el sistema de conducción Ramé, probando si su variante de doble planta es más eficiente para producir cantidad y calidad de uva que la versión de planta simple, mediante un ensayo experimental. 


\section{Material y método}

El ensayo se realizó en la temporada 2014-2015 en un viñedo localizado en el departamento de Luján de Cuyo, perteneciente a la parcela experimental de la Cátedra de Viticultura de la Facultad de Ciencias Agrarias, UNCuyo, Mendoza.

Para la descripción del sitio se tomaron los datos de la Estación Agrometeorológica Chacras de Coria, de la Cátedra de Meteorología Agrícola de la Fac. de Cs. Agrarias de la U.N.Cuyo, ubicada en el Departamento Luján de Cuyo a los 32059' de latitud Sur, 685' de longitud Oeste y 920 msnm, a 200 metros del ensayo.

En el sistema de conducción Ramé se estableció un ensayo experimental con diseño estadístico, con dos tratamientos. Un tratamiento fue colocar una planta por palo, al cual le llamamos Ramé simple (RS) y otro de 2 plantas por palo, al cual le llamamos Ramé doble (RD), quedando de esta manera distinto número de plantas por unidad de superficie.

\subsection{Mediciones realizadas}

Superficie foliar expuesta potencial (SFEm2/Ha): Se realizó en envero, con el método geométrico propuesto por Carbonneau (Carbonneau, 1985). Producción por planta: Se realizó la cosecha de cada una de las plantas muestreadas, en forma individual, para poder tener el peso individual por planta. La cosecha se realizó en cajas para cosecha

Variables cualitativas:

a) Azúcar: por refractómetro en campo y densímetro en laboratorio.

b) Acidez total: por titulación.

c) Antocianos: técnica generada por el laboratorio de polifenoles del INTA basada en la técnica de Riou y Asselin 1996.

d) Polifenoles totales: técnica generada en el laboratorio de polifenoles del INTA basada en la técnica de Riou y Asselin 1996.

\section{Resultados}

Dentro de las variables estudiadas, presentamos en este trabajo las variables de Superficie foliar total por unidad de producción (SFTr/P), Antocianos (mg/Kg de uva) e Índice de polifenoles totales (IPT).

Como se puede ver en la Tabla 1, no se observan diferencias significativas en ninguna de las tres variables estudiadas, (SFTr/P), Antocianos e IPT.
Tabla 1. Resultados de las variables de superficie foliar total por producción de uva, Antocianos e IPT.

\begin{tabular}{|l|l|l|}
\hline Variable & RS & RD \\
\hline SFTr/P & $0,53 \mathrm{~A}$ & $0,60 \mathrm{~A}$ \\
\hline Antocianos mg/ kg uva & $1,19 \mathrm{~A}$ & $1,22 \mathrm{~A}$ \\
\hline $\begin{array}{l}\text { IPT (fenoles totales de los } \\
\text { hollejo }\end{array}$ & $42,67 \mathrm{~A}$ & $43,00 \mathrm{~A}$ \\
\hline $\begin{array}{l}\text { Letras iguales indican que no hay diferencia significativa } \\
\text { para p menor que 0,05 }\end{array}$ \\
\hline
\end{tabular}

En cuanto a la SFTr/P, puede observarse que toma valores entre 0,5 y 0,6 , similares a valores encontrados por Carbonneau y otros autores para sistemas de canopia dividida en dos planos de vegetación vertical.

Los datos de variables cualitativas como Antocianos e IPT, alcanza valores de alta calidad enológica, descriptos por distintos autores como Cargnello (2003), Baeza (2005), Carbonneau (2007, 2010).

\section{Conclusiones}

El sistema Rame se presenta como un buena alternativa para zonas con alta oferta energéticas, al poder dividir la canopia en dos planos de vegetación, lo que permite una mayor superficie foliar y una alta concentración de polifenoles.

A su vez, el poder mecanizar la cosecha, facilita una labor que es bastante compleja en nuestra región.

\section{Referencias}

Baeza, C.R., Encarna C., Vicente S., and Lissaguirre J.R. 2005. Ecophysiological and Agronomic Response of Tempranillo Grapevines to Four Training System. Am. J. Enil. Vitic. 56:2

Bergqvist, J., Dokoozlian N. and Ebisuda N. 2000. Sunlight exposure and temperature effects on berry growth and composition of cabernet sauvignon and Grenache in the central san Joaquin valley of California. Am. J. Enol. Vitic. 52:1

Carbonneau, A. 1985. Trellising and canopy management for cool climate viticulture. Proc. First Int.Symp. Cool climate viticulture and Enology, June, 1984 Eugene, Oregon State University, 158-174

Carbonneau, A. 1995. La surface foliaire exposée pontentielle. Guide pour sa mesure. Pregr. Agric. Vitic., 112 (9): 204-212

Carbonneau, A. 2007. Théorie de la maduratión et de la typicité du raisin. Progr. Agric.Vitic. (Comité de lecture), et CR GESCO 15, 123 (13-14), 275-284 